\title{
X-ray Morphology of Clusters of Galaxies and its Application
}

\author{
Y. Hashimoto ${ }^{1}$, H. Boehringer ${ }^{1}$, J. P. Henry ${ }^{2}$, G. Hasinger ${ }^{1}$, \\ and G. Szokoly ${ }^{1}$ \\ ${ }^{1}$ Max-Planck-Isntitut fuer extraterestrische Physik, Garching, Germany email: \\ hashimot@mpe.mpg.de \\ ${ }^{2}$ Institute of Astronomy, University of Hawaii 2680 Woodlawn Dr. Honolulu, HI 96822, USA
}

Abstract. We report our study of X-ray cluster morphology using a sample of 101 clusters of galaxies at redshift $\mathrm{z} \sim 0.05-1$ taken from the Chandra archive.

Keywords. galaxies: clusters: general, X-rays: galaxies: clusters

\section{Results}

The X-ray morphology is quantitatively characterized by a series of objectively measured simple statistics (concentration, asymmetry, ellipticity, and offcenter-ness) of the X-ray surface brightness distribution, which are designed to be robust against variations of image quality caused by various exposure times and various cluster redshifts. Using these measures, we quantitatively investigated the relationships between the cluster Xray morphology and various other cluster characteristics. Our main results are: (1) The distorted and non-distorted clusters occupy well-defined loci in the L-T plane, demonstrating the measurements of the global luminosity and temperature for distorted clusters should be interpreted with caution, or alternatively, a rigorous morphological characterization is necessary when we use a sample of clusters with heterogeneous morphological characteristics to investigate the L-T or other related scaling relations. (2) Ellipticity and Off-center show no evolutionary effects between high and low redshift cluster subsets, while there may be a hint of weak evolutions for the Concentration and Asymmetry, in such a way that high-z clusters show more distorted morphology.
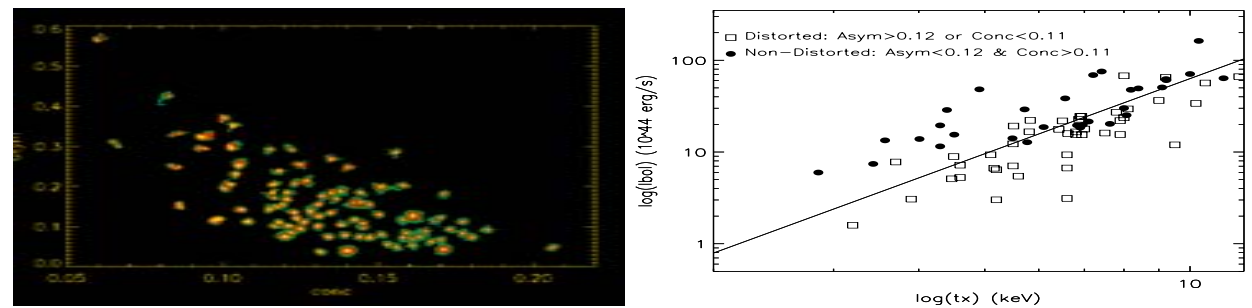

Figure 1. Left: Distributions of cluster X-ray morphology in the Conc-Asym plane. Each cluster point is represented by a X-ray image of the cluster. Right: Cluster X-ray morphology in the L-T plane. The solid line is the fitted line by Wu et al. (1999). The solid ovals are the "non-distorted" clusters and open rectangles are the "distorted" clusters.

\section{Reference}

Hashimoto, Y., Boehringer, H., Henry, J.P. et al. 2006, ApJ (in press). 